
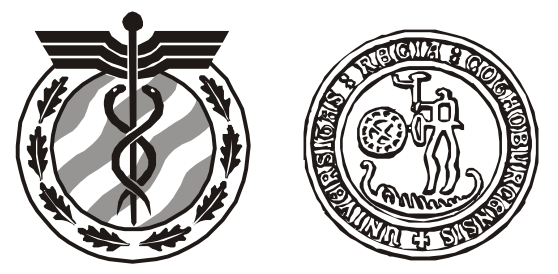

Research Report

Statistical Research Unit

Department of Economics

University of Gothenburg

Sweden

\title{
Methods and evaluations for surveillance in industry, business, finance, and public health
}

\section{Frisén, M}

Research Report

2011:3

ISSN 0349-8034

Mailing address:

Statistical Research Unit

P.O. Box 640

Fax

Nat: 031-786 1274

Int: +46317861274
Phone

Nat: 031-786 0000

Int: +46317860000
Home Page:

http://www.statistics.gu.se/

SE 40530 Göteborg

Sweden 


\title{
Methods and evaluations for surveillance
}

\section{in industry, business, finance, and public health}

\author{
Marianne Frisén \\ Statistical Research Unit, Department of Economics, University of Gothenburg, SE40530 \\ Gothenburg, Sweden
}

An overview on surveillance in different areas is given. Even though methods have been developed under different scientific cultures, the statistical concepts can be the same. When the statistical problems are the same, progress in one area can be used also in other areas. The aim of surveillance is to detect an important change in an underlying process as soon as possible after the change has occurred. In practice, we have complexities such as gradual changes and multivariate settings. Approaches to handling some of these complexities are discussed. The correspondence between the measures for evaluation and the aims of the application is important. Thus, the choice of evaluation measure deserves attention. The commonly used ARL criterion should be used with care.

Keywords: expected delay; gradual change; likelihood ratio; monitoring; multivariate surveillance

\section{INTRODUCTION}

Statistical methods play an important role to assure quality and reliability in industry ${ }^{1}$. Industrial quality control requires material checks, monitoring of the production and assembly process as well as control of the finished goods. The different certificates issued for companies with good quality control are important marketing tools. For some purposes retrospective analyses are useful, but here on-line surveillance, aimed at detecting a serious change, will be discussed. The theory and application of statistical surveillance started in industrial production. Around 1930, Walter A. Shewhart developed the first versions of sequential surveillance by introducing control charts for industrial applications ${ }^{2}$, and methods are still being developed in that area. Complex problems will be discussed in Section 5. One example is the multivariate problem of monitoring several sources of variation in the assembly process of the Saab automobile ${ }^{3}$. In business, timely decisions are important. If, for example, the churn rates change ${ }^{4}$ it should be detected as soon as possible. For business decisions it is also important to timely predict the shift from a period of expansion to one of recession. Leading economic indicators can be used to predict the turns of business cycles ${ }^{5,6}$. The changes are complex, and in Section 5 nonparametric maximum likelihood estimates will be discussed as a basis for a surveillance system.

The textbooks on quality control by Montgomery ${ }^{7}$ and Ryan $^{8}$ and overviews for example by Woodall and Montgomery ${ }^{9}$ focus on quality control in industry. Surveys on statistical surveillance are given for example by $\mathrm{Lai}^{10}$, who gives a full treatment of the field but concentrates on the minimax properties of stopping rules, and by Frisén ${ }^{11}$, who characterizes methods by their different optimality properties. The terminology is diverse. "Optimal stopping rules" is most often used in connection with financial problems when full knowledge about 
the model is assumed, so that probability theory can be used to determine the optimal time for trading. Literature on "change-point problems" does not always treat the case of sequentially obtained observations but sometimes refers to the retrospective analysis. The term "early warning system" is sometimes used in economic and medical literature. The term "monitoring" is most often used in medical literature and with a broad meaning. In the literature on industrial production, the terms "statistical process control" (SPC) and "quality control" are used. These concepts are often used in a broad sense.

The aim of sequential surveillance is the timely detection of important changes in the process that generates the data. The general questions that are always inherent in surveillance inference are treated in Section 2. The inferential aims of surveillance differ from those of hypothesis testing. Since the aims are different, so are also the evaluations and methods which meet these aims. In Section 3, some commonly used optimality criteria are described, and general methods to aggregate information sequentially in order to optimize surveillance are discussed.

One of the stated aims of the European Network for Business and Industrial Statistics, ENBIS, is to facilitate the rapid transfer of statistical methods and related technologies to and from business and industry. In Section 4, some important applications in finance and public health are described to demonstrate inferential similarities to and differences from those used in industry and business. Besides the important business and industrial applications, many new applications have come into focus. Even though the methods have been developed under different scientific cultures, inferential similarities can be identified. The description of some important applications in Section 4 also serves to demonstrate some general types of complexity for which special developments are needed. Emerging needs in other areas and the availability of powerful computing resources have encouraged the development of more advanced and efficient methods that take newer optimality requirements into account. General approaches to constructing methods for such situations are discussed in Section 5. The discussion in Section 6 contains some reflections on the future of the theory and applications of statistical surveillance.

\section{CHARACTERISTICS OF SURVEILLANCE}

In this section, we treat the basic characteristics of all on-line surveillance. Some complex problems of surveillance connected to the applications in Section 4 will be treated in Section 5 .

In on-line surveillance we follow a process sequentially and make repeated observations. We also make sequential decisions. Neither of these characteristics distinguishes surveillance from sequential hypothesis testing. However, in hypothesis testing there is a fixed hypothesis, and we just gather more information about whether this hypothesis is true or not. This is not the case in surveillance. The monitored process may work well at first, but after some time the machine may break down so that there is a change during the observation period. We cannot accept the null hypothesis and stop the monitoring even if the process is fine for a long time. The machine may start to produce faulty items after some time, and we want to detect that. Another specific characteristic of surveillance is that time is important. We need timely decisions. To retrospectively test whether there was a change is something completely different. In surveillance we need to determine, at each decision time, whether we have enough information or if we should wait for more observations until we give an alarm.

In Section 2.1, notations are introduced and the statistical surveillance problem is specified. The choice of evaluation measures should match the aims of the surveillance, as it will decide which methods are considered appropriate. Thus, the metrics for evaluation are impor- 
tant and closely related to the character of the surveillance. Evaluation in surveillance is described in Section 2.2.

\subsection{Notations and specifications}

The variable under surveillance could be a direct observation, like the measurement of the length of a produced item, or a derived statistic, like the autocorrelation between successive measurements, or even a vector of observations. We denote the process by $X=\{X(t): t=1$, $2, \ldots\}$, where $\mathrm{X}(\mathrm{t})$ is the observation (vector) made at time $\mathrm{t}$. We consider discrete time.

The purpose of the monitoring is to detect a possible change, for example in the average length. The time for the change is denoted by $\tau$. In this section as well as the next one, we consider only one change. Methods for the more complex problems in Section 4 are described in Section 5. Before the change, the distribution belongs to one family, $\mathrm{f}^{\mathrm{D}}$, and after the change at time $\tau$, it belongs to another family, $\mathrm{f}^{\mathrm{C}}$. At each decision time $\mathrm{s}$, we want to discriminate between two events, $\mathrm{C}(\mathrm{s})$ and $\mathrm{D}(\mathrm{s})$. For most applications, these can be further specified as $C(s)=\{\tau \leq s\}$ (at the decision time, there has been a change) and $D(s)=\{\tau>s\}$ (at the decision time, no change has occurred yet), respectively. The (possibly random) process that determines the state of the system could, for example, be a parameter in the distribution. Most studies in literature concern a step change, and this will be considered in this and the next section, while methods for more complex problems, which are needed for the applications in Section 4, will be treated in Section 5.

The alarm statistic, $\mathrm{p}\left(\mathrm{X}_{\mathrm{s}}\right)$, should be based on the observations $X_{\mathrm{s}}=\{\mathrm{X}(\mathrm{t}) ; \mathrm{t} \leq \mathrm{s}\}$ available at the decision time $\mathrm{s}$. The distribution of the time of the alarm, $t_{A}$, is dependent on the alarm statistic and a control limit, G(s), as

$$
\mathrm{t}_{\mathrm{A}}=\min \left\{\mathrm{s} ; \mathrm{p}\left(\mathrm{X}_{\mathrm{s}}\right)>\mathrm{G}(\mathrm{s})\right\} \text {. }
$$

Often the surveillance is active, as the process is immediately stopped or changed at the alarm (for example in order to adjust a machine in industrial production). In some applications, by contrast, surveillance is passive ${ }^{12}$ in the respect that the process is not affected by the alarm. In the automatic monitoring of disease incidences, the incidence will not be immediately affected by the detection of an unusual value. In passive surveillance, the error risks are different from those in active surveillance ${ }^{13,12}$. There are some recent suggestions of special evaluation metrics for passive surveillance in public health ${ }^{14,15,16}$. However, the first alarm has a special meaning also in passive surveillance. Here, only the properties up to the first alarm will be considered.

The change point $\tau$ can be regarded either as a random variable or as a deterministic but unknown value, depending on what is most relevant for the application. If the time $\tau$ is regarded as a random variable, we have a distribution. This can be regarded as a prior distribution if Bayesian inference is used. The intensity, $v(t)$, of a change is defined as $v(t)=P(\tau=t \mid \tau \geq t)$, which is often assumed to be constant over time. The same methods can be derived by Bayesian or frequentistic inference. However, the evaluations differ. Within the Bayesian framework all information is contained in the posterior distribution, but in this paper frequentistic inference is used. This calls for other measures, as will be seen in the next section.

\subsection{Evaluation measures}

The evaluation measures are important since they should correspond to the aims . Quick detection and few false alarms are desired properties of methods for surveillance. For some applications, also other specific requirements are relevant. These questions are discussed in Section 4. 
Evaluation by significance level, power, specificity, sensitivity, or other well-known metrics may seem convenient. However, these metrics are not easily interpreted in a surveillance situation since they change with the length of the surveillance. The significance level has no unique value for methods commonly used in a surveillance system. The probability of a false alarm will tend to one when the length of the surveillance tends to infinity. The problems regarding the use of the conventional metric significance level apply also to power, specificity, and sensitivity. Accordingly, conventional measures should be supplemented by other measures designed for statistical surveillance.

\subsubsection{False alarms}

The most commonly used measure for surveillance is the Average Run Length when there is no change, $A R L^{0}=E\left(t_{A} \mid D\right)$. A variant of the ARL is the Median Run Length, MRL, which is convenient to use in simulation studies. A measure commonly used in theoretical work is the probability that the alarm occurs before the change, $\mathrm{PFA}=\mathrm{P}\left(\mathrm{t}_{\mathrm{A}}<\tau\right)$. The distribution of $\tau$ is also involved here. Other measures of the false alarm tendency have also been suggested in connection with complex situations and special applications.

\subsubsection{Delay}

The most commonly used measure of the delay is the ARL ${ }^{1}$, which is the Average Run Length until the detection of a change (if the change occurred at the same time as the surveillance started). The part of the definition within parentheses is not always spelled out but generally used ${ }^{17,8}$.

An alternative measure of delay, which is closely related to a highly general utility func$\operatorname{tion}^{18}$, is the expected value of the delay from the time of change, $\tau=t$, to the time of alarm, $t_{\mathrm{A}}$. It is here denoted by

$$
\mathrm{ED}(\mathrm{t})=\mathrm{E}\left[\max \left(0, \mathrm{t}_{\mathrm{A}}-\mathrm{t}\right) \mid \tau=\mathrm{t}\right] .
$$

Since the value of $\operatorname{ED}(\mathrm{t})$ will typically tend to zero as $\mathrm{t}$ increases, it may be preferable to use the conditional expected delay

$$
\mathrm{CED}(\mathrm{t})=\mathrm{E}\left[\mathrm{t}_{\mathrm{A}}-\tau \mid \mathrm{t}_{\mathrm{A}} \geq \tau=\mathrm{t}\right]=\mathrm{ED}(\mathrm{t}) / \mathrm{P}\left(\mathrm{t}_{\mathrm{A}} \geq \mathrm{t}\right) \text {. }
$$

Note that $\mathrm{ARL}^{1}=\mathrm{CED}(1)+1=\mathrm{ED}(1)+1$. For most methods, the $\mathrm{CED}(\mathrm{t})$ will converge to a constant value when the time of the change increases. This value is the Steady state Average Delay Time, $\mathrm{SADT}^{19,20}$. It is, in a sense, the opposite of $\mathrm{ARL}^{1}$ since only very large values of $\tau$ are considered.

The first ones to use the notation CED and calculate CED for a specific value of $\tau$ seem to have been Zacks and Kenett in $1994^{21}$. The present author advocates ${ }^{11,22}$ that CED be calculated for several values of $\tau$ to give the whole picture.

Sometimes, the time available for rescue actions is limited. The Probability of Successful Detection ${ }^{23}$ measures the probability of detection with a delay time no longer than a constant d

$$
\operatorname{PSD}(\mathrm{d}, \mathrm{t})=\mathrm{P}\left(\mathrm{t}_{\mathrm{A}}-\tau \leq \mathrm{d} \mid \mathrm{t}_{\mathrm{A}} \geq \tau=\mathrm{t}\right) .
$$

It may be useful to describe the ability to detect the change within a certain time also when there is no absolute detection time limit. The PSD can be calculated for different time limits $^{24,15}$. The ability to make a very quick detection (small d) is important in surveillance of sudden major changes, while the long-term detection ability (large d) is more important in surveillance where smaller changes are expected. 


\subsubsection{Predictive value}

When there is an alarm, it is important to know whether this alarm is a strong indication of a change or just a weak one. To judge this, it is necessary to consider the risk of false alarms, the expected delay, and how often changes occur. If $\tau$ is regarded as a random variable, this can be done by one summarizing measure. The predictive value of an alarm was suggested ${ }^{23}$ as

$$
P V(t)=P\left(\tau \leq t_{A} \mid t_{A}=t\right) .
$$

This is the probability that a change has occurred when there is an alarm. Some methods have a nearly constant PV. Others, like the Shewhart method, have a very low PV at early alarms but a higher one later (for a constant intensity $v$ ). The early alarms will not prompt the same serious action as later ones. In fact, one might consider disregarding early alarms by some methods. The FIR variants ${ }^{25}$, which give a Fast Initial Response, have the opposite aim and are suitable when the probability of an immediate change is very high.

By choosing an alarm limit which results in a good predicted value, a relevant balance between the false alarms and the delay may be obtained. A computer program which illustrates the balance between delay and false alarms by the predicted value for different situations and methods is available for download at www.statistics.gu.se/surveillance, where also other free computer programs for surveillance can be found.

\section{OPTIMAL METHODS}

Different methods for aggregating information over time will be suitable for different situations. In order to see the correspondence we will first, in Section 3.1, specify commonly used optimality criteria and then, in Section 3.2, describe the methods which are optimal according to these different criteria.

\subsection{Optimality criteria}

The delay of an alarm depends on whether the change appears early or late after the start of the surveillance. In addition, this dependency is different for different methods. There is thus no single simple summarizing measure. Instead, several optimality criteria have been suggested.

\subsubsection{ARL optimality}

The most commonly used optimality criterion is stated as minimal $\mathrm{ARL}^{1}$ for a fixed $\mathrm{ARL}^{0}$. $\mathrm{ARL}^{1}$ is the expected run length under the assumption that $\tau=1$ and that the observations at all time points have distributions which belong to $\mathrm{f}^{\mathrm{C}}$. $\mathrm{ARL}^{0}$ is the expected value of the run length given that all observations have distributions which belong to $\mathrm{f}^{\mathrm{D}}$. Discriminating between the two alternatives that all observations come from either of the two distributions should allocate the same weight to all observations by the ancillary principle. However, efficient methods for surveillance will give most weight to the most recent observations. One should beware when violating inference principles. The dominating position of the ARL criterion was questioned ${ }^{11}$ as some (artificial) methods which are useless in practice are ARL optimal.

An argument for the ARL criterion has been ${ }^{26}$ that for many methods it agrees with a variant of the minimax criterion. However, it was demonstrated ${ }^{22}$ that for the EWMA method (see Section 3.2.5), there is no similarity between the optimal parameter values according to the ARL criterion and those according to the minimax criterion, while the optimal parameter 
values by the criterion of expected delay and the minimax criterion agree well. The ARLoptimal value of $\lambda$ in the EWMA method is zero ${ }^{11}$, and it was demonstrated ${ }^{22}$ that other claims correspond to a local minimum. The ARL can be used as a descriptive measure but is questionable as a formal optimality criterion.

\subsubsection{SADT optimality}

The steady state delay time, SADT, measures the delay asymptotically when $\tau$ tends to infinity. This emphasis on late changes is thus the opposite of the ARL criterion, which focuses on early changes. In an evaluation ${ }^{27}$ of methods for surveillance of small incidence rates it was demonstrated that methods which were earlier (when judged by the ARL) considered to be the most efficient were the least efficient when judged by SADT.

\subsubsection{Minimal expected delay}

The expected delay depends on the time of the change, $\tau$. Instead of giving emphasis to extremely early (ARL) or extremely late (SADT) changes, it is natural to evaluate by an average. The minimizing of this average, for a fixed false alarm probability, is termed the ED criterion (the minimal expected delay criterion). The ED criterion corresponds to a highly general utility function ${ }^{18}$.

\subsubsection{Minimax optimality}

A minimax solution, with respect to $\tau$, avoids the requirements of information about the distribution of $\tau$. Often, an even more pessimistic criterion is used. The "worst possible case" is determined by using not only the least favorable value of the change time, $\tau$, but also the least favorable outcome of $\mathrm{X}_{\tau-1}$ before the change occurs. The minimax criterion ${ }^{28}$, upon which much theoretical research is based, is the minimum of:

$$
\sup \operatorname{ess} \sup E_{\tau}\left\{\left[t_{A}-\tau+1\right]^{+} \mid X_{\tau-1}\right\} \text {, }
$$

for a fixed $\mathrm{ARL}^{0}$.

\subsection{Methods}

For some methods, the delay is short for early changes but bad for later ones. For other methods the case is the opposite. Thus, different methods will turn out to be the best depending on which summarizing measure is used. Different optimality criteria will give different answers to the question of which method is the best for a specific situation, since different optimality criteria put emphasis on different values of $\tau$.

The sequentially obtained information will be aggregated in order to take advantage of all information. Many methods for surveillance can be expressed by a combination of partial likelihood ratios. The likelihood ratio for a fixed value of $\tau$ is

$$
\mathrm{L}(\mathrm{s}, \mathrm{t})=\mathrm{f}_{\mathrm{Xs}}\left(\mathrm{X}_{\mathrm{s}} \mid \tau=\mathrm{t}\right) / \mathrm{f}_{\mathrm{Xs}}\left(\mathrm{X}_{\mathrm{s}} \mid \mathrm{D}\right) \text {. }
$$

The formula for these likelihood components will vary between situations. Commonly used methods are often expressed for simple settings like independent Gaussian observations with a step shift. Here, generalized versions are described by the likelihood expression. This is the basis for adaptation to the more complex settings in Section 5.

\subsubsection{The full likelihood ratio method}

The full likelihood ratio method (LR) with the alarm statistic 


$$
\frac{\mathrm{f}_{X_{\mathrm{s}}}\left(\mathrm{x}_{\mathrm{s}} \mid \mathrm{C}(\mathrm{s})\right)}{\mathrm{f}_{X_{\mathrm{s}}}\left(\mathrm{x}_{\mathrm{s}} \mid \mathrm{D}(\mathrm{s})\right)} .
$$

is optimal with respect to the criterion of minimal expected delay and also to a wider class of utility functions ${ }^{12}$. The full likelihood statistic can be expressed as a weighted sum of the partial likelihoods $\mathrm{L}(\mathrm{s}, \mathrm{t})$. The weights are proportional to the density of $\tau$. An equivalent ${ }^{12}$ expression is by the posterior probability. This has motivated the name "the Bayes method." However, it depends on the application whether the distribution of $\tau$ should be considered as a "prior", as an observed frequency distribution, or just as the situation for which optimality is needed.

\subsubsection{The Shiryaev-Roberts method}

The simplest way to aggregate the likelihood components is to add the partial likelihood expressions. This means that all possible change times, up to the decision time s, are given equal weight. The method ${ }^{18,29}$, now called the Shiryaev-Roberts method, can also be given a natural interpretation if the time of the change, $\tau$, is regarded as a random variable. The method can then be regarded as a special case of the full likelihood ratio method where the intensity $v$ tends to zero. It can also be seen as the LR method with a non-informative prior for $\tau$.

\subsubsection{The Shewhart method}

This method is simple and the most commonly used method for surveillance. The alarm is given as soon as an observation deviates too much from the target. Thus, only the last observation is considered. The alarm statistic of the LR method reduces to that of the Shewhart method when we specify $C(s)=\{\tau=s\}$ and $D(s)=\{\tau>s\}$. Thus, the Shewhart method has optimal error probabilities when we want to discriminate between a change at the current time point and the case that no change has yet occurred for these alternatives. By several criteria, however, the Shewhart method performs poorly ${ }^{30}$ for small and moderate shifts.

\subsubsection{CUSUM}

The CUSUM method ${ }^{17,31}$ can be expressed by the partial likelihood ratios as

$$
t_{A}=\min \{s ; \max (L(s, t) ; t=1,2, . ., s)>G\},
$$

where $t_{A}$ is the time of the alarm and G is a constant. The CUSUM method satisfies the minimax criterion of optimality described in Section 3.1.4.

\subsubsection{Exponentially weighted moving average}

The alarm statistic of the EWMA method ${ }^{32}$ is an exponentially weighted moving average,

$$
Z_{s}=(1-\lambda) Z_{s-1}+\lambda Y(s), s=1,2, \ldots
$$

where $0<\lambda<1$ and $Z_{0}$ is the target value. The asymptotic variant, EWMAa, will give an alarm at

$$
\mathrm{t}_{\mathrm{A}}=\min \left\{\mathrm{s}: \mathrm{Z}_{\mathrm{s}}>\mathrm{L} \sigma_{\mathrm{Z}}\right\},
$$

where $\mathrm{L}$ is a constant. In the exact variant, EWMAe, the exact standard deviation is used instead of the asymptotic one in the alarm limit. EWMAe can be regarded as a repeated significance test, but the EWMAa version may be preferable in other respects ${ }^{33}$.

The EWMA statistic gives decreasing weights to earlier observations. If $\lambda=1$, the EWMA method reduces to the Shewhart method, but if $\lambda$ approaches zero all observations have ap- 
proximately the same weight. Small values of $\lambda$ result in a good ability to detect early changes, while larger values are necessary to detect later changes. The search for the optimal value of $\lambda$ has attracted much attention in literature. Most reports on optimal values of the parameter $\lambda$ refer to the ARL criterion. However, it was demonstrated ${ }^{11}$ that by this criterion, $\lambda$ should approach zero. Wisely enough, this value of $\lambda$ is seldom used in practice since it would seriously reduce the ability to detect late changes.

The EWMA method cannot be directly expressed by partial likelihood expressions, but it can be expressed as an approximation of the full LR method. The ED optimal value of $\lambda$ was derived $^{11}$, and the optimality properties were illustrated by large-scale simulation studies ${ }^{22}$.

\subsubsection{Adaptability of methods}

Some methods are flexible and have several parameters. The parameters can be adapted to make the method optimal for specific conditions, for example regarding the size of the change or the intensity of changes. The flexible methods give possibilities, but the burden to choose the parameters is sometimes seen as an argument for using less flexible methods. The LR method can be optimized both for shift size and for intensity. The EWMA method can be optimized for a combination of shift size and intensity, while the CUSUM and ShiryaevRoberts methods can be optimized for shift size. The Shewhart method does not have any parameters to optimize for. However, all the other methods tend to the Shewhart method when the size of the shift tends to infinity ${ }^{34}$.

\section{SURVEILLANCE IN FINANCE, PUBLIC HEALTH, AND OTHER AREAS}

In the past, industrial quality control dominated the development of surveillance theory. In recent years, however, much development has occurred within other areas together with a fruitful cross-fertilization between areas ${ }^{35,16}$. Two areas of different kinds and of recent interest are financial surveillance and public health surveillance. They will be described in some detail, while some other areas will be described more briefly. The aim is to illustrate the diversity of surveillance needs and to indicate inferential similarities between apparently different surveillance problems.

\subsection{Financial surveillance}

In finance, the timeliness of transaction strategies is obvious. There are many textbooks describing financial problems and statistical models and methods ${ }^{36,37,38,39,40,41,42}$. Various statistical techniques are described in these books.

When the stochastic model is completely known, we assume an efficient market and can use probability theory to calculate the optimal transaction conditions. The mathematical and probabilistic aspects of finance have developed considerably. Important contributions are found for example in the journal Finance and Stochastics.

The theory of an arbitrage-free market may seem convincing. However, there are some doubts that it is generally applicable in practice, and many efforts are made to increase the return of an investment. The efficient market hypothesis depends on the complete knowledge about the model. When the information about the process is incomplete, there may be an arbitrage opportunity ${ }^{43}$. If, for example, a change can occur in the process, observations should be analyzed continuously to decide whether a transaction at a certain time point is profitable as measured either by return or by risk. Statistical surveillance is needed for the decision ${ }^{44}$. Different aspects of the subject of financial surveillance are described in the book ${ }^{45}$.

Since financial settings are often complex, approaches for surveillance in more complicated situations than those described in the earlier sections are of interest. Advanced stochastic models are used to capture important features in finance. The expected value could depend 
on time in a complicated nonlinear way. Peaks and troughs are often of special interest as trading indicators. The detection of such events will be discussed in Section 5.1. In finance it is natural to measure the success of a transaction strategy by the return of the investment. Parameters other than the expected value are often of great interest. The risk (measured by variance), is one such example. A transaction strategy that offers a low risk, as measured by variance (volatility), is preferable. Thus, methods for surveillance of the variance ${ }^{46}$ in a financial series are of interest. Moreover, complicated dependency structures are common. Nonlinear time series models, like the GARCH model ${ }^{47}$, incorporate some of these features. Multivariate data streams are of interest for example when choosing a portfolio ${ }^{48}$ of stocks and will be discussed in Section 5.2.

\subsection{Public health surveillance}

The timely detection of various types of adverse health events is crucial ${ }^{49}$. The delay of one day in the detection of and response to an epidemic due to a bioterrorist attack can result in the loss of thousands of lives and millions of dollars ${ }^{50}$. Today different kinds of data ${ }^{51}$ are collected to monitor for bioterrorism. The need for surveillance of malpractice came into focus after the serial killings by the British family physician Harold Shipman. The monitoring of mortality rates ${ }^{52}$ in primary care was partly motivated by this case.

The monitoring of incidences of different diseases and symptoms is carried out by authorities to detect outbreaks of infectious diseases. Epidemics, such as influenza, are for several reasons very costly to society, and it is therefore of great value to monitor both for the outbreak and during the epidemic period in order to allocate medical resources. Methods for the surveillance of common diseases can also serve to detect new ones. The models of influenza for surveillance purposes are complex ${ }^{53}$. Gradual changes from unknown baselines are common in the area of public health and will be discussed in Section 5.1.2. The detection of both the onset ${ }^{54}$ and the peak ${ }^{55}$ of the epidemic period requires methods which are robust ${ }^{56}$. Predictions of the characteristics of the present influenza period from early observations are important for the planning of health resources ${ }^{57}$.

Most of the theory of surveillance is derived for normal distributions, but in public health other kinds of distributions are of interest. Poisson processes are of special interest in public health surveillance where an increased incidence of adverse health events is serious. The need for systems for the detection of an increased birth rate of babies with congenital malformations $^{58,59}$ was apparent after the thalidomide tragedy in the early 1960s. Much suffering could have been avoided if the harm of the medication had been detected earlier. The distance between negative events, measured by the number of positive ones in-between, can be used when no other time scale is relevant. There are methods designed for such a case, where the negative event is the birth of a baby with congenital malformation and the positive one is the birth of a healthy baby ${ }^{27}$.

The detection of a clustering of cases may reveal a source of adverse health events ${ }^{60}$. Spatial surveillance is frequently used in the area of public health, partly because of the effective and freely available computer programs by Kulldorff ${ }^{61}$. Spatial surveillance can be seen as a special case of multivariate surveillance, as discussed in Section 5.2.

In public health surveillance, quick detection is beneficial both at an individual level and to society. Recently there has been a vivid discussion of evaluation metrics. Some recent suggestions regarding evaluation in public health surveillance have been based on the requirement of simplicity coming from the medical authorities who have to handle the information in this new area. Thus, these suggestions are often based on metrics suitable for the more familiar hypothesis testing situation. However, complex problems seldom have simple solutions. 


\subsection{Monitoring of patients}

In intensive care as well as in more sparse contacts with patients, monitoring is needed to detect changes. The technical advancements and the improved recording of the status of patients should be supplemented by a statistical surveillance system. One example of surveillance in intensive care is the monitoring of the signals from the baby's heart during labor ${ }^{23}$. An example of less frequent data is the surveillance of the growth of the fetus during pregnancy. Both the individual factor of the body size of the mother and the general growth pattern will have influence on the growth. A longitudinal model was used in the derivation of likelihood based surveillance ${ }^{62}$. Surveillance of patients after a kidney transplant ${ }^{63}$ was carried out by a fully Bayesian inference.

\subsection{Environment monitoring}

There is a growing interest in detecting changes in the environment. Needs for environmental control are described for example in the journal Environmetrics. The dependency structure is important for a system for the surveillance of biodiversity ${ }^{64}$. In 1986 there was the nuclear accident at Chernobyl in the former Soviet Union. Later in the same year, the Swedish Radiation Protection Institute installed 37 stations for measuring radiation. Likelihood based surveillance with modeling of the background radiation was used in a surveillance system ${ }^{65,66}$.

\section{COMPLEX SITUATIONS}

Real world applications are often complex, as was seen in Section 4. Thus, the basic surveillance theory described in Sections 2 and 3 has to be adapted to special issues. There are many different complexities of interest.

The theory of surveillance of dependent data over time, such as autocorrelated time series, is not simple due to the inherent time structure of surveillance. The most common approach to the surveillance of models with time dependencies is to make the alarm conservative by increasing the alarm limit of an ordinary method, so that the false alarm risk is controlled $^{67}$. Another common approach is to monitor the process of residuals ${ }^{68}$. This is especially useful in complicated time series models like GARCH, where explicit likelihood expressions are not available ${ }^{47}$. The first observation in an autocorrelated series is special. There have been several suggestions about how to handle the first statistic and how to ease the computational burden ${ }^{69,70,71,72}$. The CUSUM method for models where the change is in the conditional density (given the previous observation) is asymptotically minimax optimal for some autocorrelated models ${ }^{73,74}$.

Most methods are constructed for normal distributions, but many applications require methods suited for other kinds of distributions. Since most methods can be expressed by likelihood functions (see Section 3.2), the change of the distribution in these functions will give methods which retain the optimality properties. For example, the EWMA method ${ }^{75}$ and the Shiryaev-Roberts method ${ }^{76}$ have been adjusted for the detection of a changed intensity in a Poisson process.

There are many more complexities to consider, and two of them will be discussed in more detail. Complex types of changes are discussed in Section 5.1, and multivariate problems are described in Section 5.2. 


\subsection{Complex changes}

\subsubsection{Changes between unknown levels}

Often, the change is characterized by a step shift in the expected value or another parameter of the distribution of the observations. The new parameter value after the change may be unknown. A Bayesian approach to handling unknown levels can be useful. A prior for the unknown shift size is used in the Mixture Likelihood Ratio (MLR) method ${ }^{77}$, which is a modification of the CUSUM method. By the Generalized Likelihood Ratio (GLR) approach, maximum likelihood estimates are used in likelihood based methods. The GLR approach for the CUSUM method is asymptotically optimal ${ }^{28}$ when the shift size is incompletely specified.

Knowledge about the shift size will increase the efficiency of the method. Errors in the pre-change conditions are even more influential since they will affect the false alarm rate and the trust in alarms ${ }^{5,54}$. In situations where we aim to detect an increase, we will get more false alarms if the baseline is underestimated than if the true value had been used. The opposite will happen if the baseline is overestimated. One way to avoid the problem of unknown parameters is to transform the data to a statistic that is invariant to the baseline. This can be done for example by using the deviation of each observation from the average of all previous observations $^{23,78,79}$.

\subsubsection{Gradual changes}

Most of the literature on surveillance treats the case of an abrupt step change. In many applications, however, the change is gradual, as mentioned in Section 4. Methods for linear changes with a known baseline and a known slope have been suggested ${ }^{80,81}$. A method for detecting when a drift exceeds a threshold ${ }^{82}$ has been suggested and compared with methods which required knowledge of the baseline. As expected, methods which utilized a known baseline worked well in comparison. When the knowledge on the shape of the curve is uncertain, non-parametric methods are of interest. A non-parametric method designed for the detection of a change in monotonicity avoids the problem of unknown baseline. The need to detect turning points was described with reference to business cycles in Section 1, with reference to finance in Section 4.1, and with reference to public health in Section 4.2. At the outbreak of an epidemic disease the incidence typically increases gradually ${ }^{83}$ and then possibly declines ${ }^{53}$. Both the onset of the outbreak and the turning point are important to detect.

The GLR approach, which has been suggested for unknown levels, can also be used for the situation where the timely detection of a change in monotonicity is of interest. The maximum likelihood estimation under order restrictions ${ }^{84,85}$ is used. The approach is semiparametric as it is nonparametric with respect to the curve shape but parametric since distributions belonging to the exponential family are used ${ }^{86,87}$. Variants of this technique were used to detect turns in financial trading ${ }^{44}$ and epidemics ${ }^{56,55}$. By constructing a system for early warnings of turns in one or several leading indicators of business cycles, the turning point time of the general business cycle can be determined using the same technique ${ }^{5,6}$. The properties of the semiparametric approach have been compared to those of parametric methods. If the parametric version is based on exact knowledge of the parameters, the parametric methods work best. In practice, however, the parameters are estimated, which affects the properties negatively and the nonparametric method can be preferred.

\subsection{Multivariate surveillance}

Multivariate surveillance is of interest in many areas, for example in financial settings ${ }^{47}$, as described in Section 4.1, and in public health surveillance ${ }^{88}$, as described in Section 4.2. Spatial surveillance is a special case of multivariate surveillance ${ }^{89}$. The surveillance of several 
distribution parameters, such as the mean and the variance ${ }^{90}$, has the same structure as multivariate surveillance. Introductions can be found in a textbook ${ }^{91}$ on multivariate quality control and in an overview on multivariate statistical process control charts ${ }^{92}$.

A natural first approach is the reduction of the dimensionality of the problem. Principal components are often used to reduce dimensionality ${ }^{93}$ and are useful when these components have a natural interpretation in the application. The most far-going dimension reduction is to reduce the information to a univariate statistic and then monitor this statistic. This is probably the most common way to handle multivariate surveillance, and if the changes occur at the same time in all variables it is optimal. This is the case, for example, at a fixture failure in a Saab automobile ${ }^{3}$. If there is a common change point $\tau$ for all variables, a sufficient reduc$\operatorname{tion}^{94}$ exists. Hence, it is possible to use the results of Section 3.2 directly. If this sufficient statistic is used in an optimal univariate method, then we have an optimal method for the multivariate problem. We can derive the likelihood components $\mathrm{L}(\mathrm{s}, \mathrm{t})$ and aggregate them by a method which guarantees optimality according to Section 3.2.l.

However, if the changes do not occur at the same time, the situation is different. Then we have a genuinely multivariate situation, and the relation between the different change points, $\tau_{1}, \ldots \tau_{p}$, for the $\mathrm{p}$ different variables is crucial. There are results on sufficient reduction ${ }^{95}$ also for the case of different change points. These results were used to construct a method for the spatial surveillance of influenza in Sweden ${ }^{96}$. A commonly used approach is the parallel surveillance of each variable, which triggers a general alarm when there is an alarm for any of the components. This works very well provided that the changes occur far apart. If the changes occur nearly simultaneously one would expect the optimal method for simultaneous changes (as discussed above) to work well. An approach between these two is a mix of the reduction by time and the reduction by variable described above. Then, the accumulated information on each component is used in a vector of component-wise alarm statistics. At each decision time, this vector is transformed into a scalar alarm statistic which is monitored. In the MEWMA method ${ }^{97,98}$, EWMA is used to accumulate the information in the first step while the Hotelling $\mathrm{T}^{2}$ control chart is used in the second step.

$\mathrm{ARL}^{1}$ is the most commonly used evaluation measure also in multivariate surveillance. By the ARL, the evaluation is made for changes occurring at the same time $\left(\tau_{1}=\tau_{2}=\ldots=\tau_{p}=1\right)$. However, the case of simultaneous changes is very special. If the changes are simultaneous, a sufficient reduction exists ${ }^{94}$. Thus, an optimal method for the multivariate surveillance problem can be constructed by the general approaches for univariate surveillance described in Section 3.2, and other methods, such as MEWMA, should not be considered. Since the ARL assumes simultaneous changes, it is not suitable for evaluating methods designed for genuinely multivariate situations with possibly different change points.

In the case of different change points, $\tau_{1}, \ldots \tau_{p}$, for the $\mathrm{p}$ different variables, the detection ability depends on when the different changes occur. Hence, we need special evaluation measures suitable for multivariate surveillance ${ }^{99}$. In many applications, it is the first change which is the most important and for which an alarm is needed. In these cases, we can concentrate on the delay from this time $\tau_{\min }=\min \left\{\tau_{1}, \ldots \tau_{p}\right\}$. The conditional expected delay in Section 2.2.2 can be generalized to

$$
\operatorname{CED}\left(\tau_{1}, \ldots \tau_{\mathrm{p}}\right)=\mathrm{E}\left(\mathrm{t}_{\mathrm{A}}-\tau_{\min } \mid \mathrm{t}_{\mathrm{A}} \geq \tau_{\min }\right) .
$$




\section{DISCUSSION}

The area of industrial quality control dominated the development of surveillance theory for a long time. In recent years, the need for surveillance in other areas has become obvious. In the last decade, the threats of bioterrorism and new contagious diseases have been important reasons behind the intensified research in the field of surveillance theory.

Walter Shewhart made pioneering work in the 1920s by introducing statistical surveillance in industrial quality control. By the Shewhart method, each observation is judged separately. Earlier, the focus was on simple standardized methods. The next important step was taken with the introduction of aggregation of information over time by the CUSUM method $^{17}$. Shortly afterwards, the EWMA method was suggested ${ }^{32}$. The full likelihood method $^{18}$, which fulfills important optimality conditions, was suggested some years later.

As has been seen, surveillance is used in more and more areas of life. These are often complex, which makes advanced statistical theory necessary. The advanced theory of surveillance, which is needed for many applications, is relatively new territory. The theory of statistical surveillance can be expected to be further developed in response to the demands of applications in various fields. A cross-fertilization back to the applications could then be expected.

The recognition that different applications have different evaluation requirements will stimulate further development of these metrics. In the past, simple evaluation techniques suitable for simple applications were dominating. Today we do no longer need evaluation techniques which are simple to compute. Evaluation methods directed at the essence of the application may be easier to explain even though the numerical computation by the computer is difficult. Robust methods, which do not require unnecessary assumptions, will be derived. Multivariate problems require further theoretical efforts.

A very rapid development of practice and research may be expected in the future. Efficient computers and computer programs will play an important role in this development. The automatic collection of different kinds of data and the new possibilities of handling large data sets constitute a good base for surveillance systems.

\section{Acknowledgement}

This work was partially supported by the Swedish Emergency Management Agency (grant 0314/206).

\section{References}

1. Ruggeri F, Kenett R, Faltin F (eds). Encyclopedia of statistics in quality and reliability. Wiley: NY, 2007.

2. Shewhart WA. Economic Control of Quality of Manufactured Product. MacMillan and Co.: London, 1931.

3. Wärmefjord K. Multivariate quality control and Diagnosis of Sources of Variation in Assembled Products. University of Gothenburg: Gothenburg, 2004.

4. Pettersson M. SPC with Applications to Churn Management. Quality and Reliability Engineering International 2004; 20:397-406.

5. Andersson E, Bock D, Frisén M. Statistical Surveillance of Cyclical Processes with Application to Turns in Business Cycles. Journal of Forecasting 2005; 24:465-490.

6. Andersson E, Bock D, Frisén M. Some statistical aspects on methods for detection of turning points in business cycles. Journal of Applied Statistics 2006; 33:257-278.

7. Montgomery DC. Introduction to statistical quality control. 2001.

8. Ryan TP. Statistical methods for quality improvement. (2nd edn). John Wiley \& Sons: New York, 2000.

9. Woodall WH, Montgomery DC. Research Issues and Ideas in Statistical Process Control. Journal of Quality Technology 1999; 31:376-386.

10. Lai TL. Sequential Changepoint Detection in Quality-Control and Dynamical Systems. Journal of the Royal Statistical Society B 1995; 57:613-658.

11. Frisén M. Statistical surveillance. Optimality and methods. International Statistical Review 2003; 71:403-434.

12. Frisén M, de Maré J. Optimal Surveillance. Biometrika 1991; 78:271-280. 
13. Kenett R, Pollak M. On Sequential Detection of a Shift in the Probability of a Rare Event. Journal of the American Statistical Association 1983; 78:389-395.

14. Kleinman K, Abrams A. Assessing surveillance using sensitivity, specificity, and timeliness. Statistical Methods in Medical Research 2006; 15:445-464.

15. Buckeridge DL, Burkom H, Campbell M, Hogan WR, Moore AW. Algorithms for rapid outbreak detection: a research synthesis. Journal of Biomedical Informatics 2005; 38:99-113.

16. Woodall WH, Marshall JB, Joner JMD, Fraker SE, Abdel-Salam A-SG. On the use and evaluation of prospective scan methods for health-related surveillance. Journal of the Royal Statistical Society A 2008; 171:223-237.

17. Page ES. Continuous inspection schemes. Biometrika 1954; 41:100-114.

18. Shiryaev AN. On optimum methods in quickest detection problems. Theory of Probability and Its Applications 1963; 8:22-46.

19. Srivastava MS, Wu Y. Comparison of EWMA, CUSUM and Shiryaev-Roberts Procedures for Detecting a Shift in the Mean. The Annals of Statistics 1993; 21:645-670.

20. Knoth S. The art of evaluating monitoring schemes - how to measure the performance of control charts? In Frontiers of Statistical Quality Control, Lenz H-J, Wilrich P-T (eds). Physica Verlag: Warsaw, 2006.

21. Zacks S, Kenett RS. Process tracking of time series with change points, . ,pp. , , , 1994. In Recent Adv. in Stat. and Prob, Vilaplana JP, Puri ML (eds). International Science Publishers, Zeist, The Netherlands, 1994.

22. Frisén M, Sonesson C. Optimal surveillance based on exponentially weighted moving averages. Sequential Analysis 2006; 25:379-403.

23. Frisén M. Evaluations of Methods for Statistical Surveillance. Statistics in Medicine 1992; 11:1489-1502.

24. Marshall C, Best N, Bottle A, Aylin P. Statistical issues in the prospective monitoring of health outcomes across multiple units. Journal of the Royal Statistical Society A 2004; 167:541-559.

25. Lucas JM, Crosier RB. Fast initial response for cusum quality control schemes: give your cusum a head start. Technometrics 1982; 24:199-205.

26. Pollak M, Siegmund D. A diffusion process and its applications to detecting a change in the drift of Brownian motion. Biometrika 1985; 72:267-280.

27. Sego LH, Woodall WH, Reynolds MR, Jr. A comparison of surveillance methods for small incidence rates. Statistics in Medicine 2008; 27:1225-1247.

28. Lorden G. Procedures for reacting to a change in distribution. Annals of Mathematical Statistics 1971; 42:1897-1908.

29. Roberts SW. A Comparison of some Control Chart Procedures. Technometrics 1966; 8:411-430.

30. Frisén M. Properties and Use of the Shewhart Method and Followers. Sequential Analysis 2007; 26:171-193.

31. Hawkins DM, Olwell DH. Cumulative Sum Charts and Charting for Quality Improvement. Springer: New York, 1998.

32. Roberts SW. Control Chart Tests Based on Geometric Moving Averages. Technometrics 1959; 1:239-250.

33. Sonesson C. Evaluations of Some Exponentially Weighted Moving Average Methods. Journal of Applied Statistics 2003; 30:1115-1133.

34. Frisén M, Wessman P. Evaluations of likelihood ratio methods for surveillance. Differences and robustness. Communications in Statistics. Simulation and Computation 1999; 28:597-622.

35. Woodall WH. The Use of Control Charts in Health-Care Monitoring and Public-Health Surveillance. Journal of Quality Technology 2006; 38:89-134.

36. Föllmer H, Schied A. Stochastic Finance. An Introduction in Discrete Time. de Gruyter: Berlin, 2002.

37. Härdle W, Kleinow T, Stahl G (eds). Applied Quantitative Finance. Theory and Computational Tools. Springer Verlag: New York, 2002.

38. Gourieroux C, Jasiak J. Financial Econometrics Problems, Models and Methods. University Presses of California, Columbia and Princeton: New Jersey, 2002.

39. Franke J, Härdle W, Hafner C. Statistics of financial markets. An introduction. Springer-Verlag: Berlin, 2004.

40. Cizek P, Härdle W, Weron R (eds). Statistical Tools for Finance and Insurance. Springer: New York, 2005.

41. Scherer B, Martin RD. Introduction to modern portfolio optimization with NuOPT ${ }^{t m}, S-P L U S$ and $S^{+}$Bayes $^{t m}$. Springer Verlag: New York, 2005.

42. Shiryaev AN. Essentials of stochastic finance: facts, models, theory. World Scientific: Singapore, 1999.

43. Shiryaev AN. Quickest Detection Problems in the Technical Analysis of Financial Data. In Mathematical Finance Bachelier Congress 2000, Geman H, Madan D, Pliska S, Vorst T (eds). Springer: Berlin, 2002.

44. Bock D, Andersson E, Frisén M. The relation between statistical surveillance and technical analysis in finance. In Financial surveillance, Frisén M (ed). Wiley: Chichester, 2007.

45. Frisén M (ed) Financial Surveillance. Wiley: Chichester, 2007.

46. Bock D. Evaluations of likelihood-based surveillance of volatility. In Financial surveillance, Frisén M (ed). Wiley: Chichester, 2007.

47. Okhrin Y, Schmid W. Surveillance of Univariate and Multivariate Nonlinear Time Series. In Financial surveillance, Frisén M (ed). Wiley: Chichester, 2007.

48. Golosnoy V, Schmid W, Okhrin I. Sequential Monitoring of Optimal PortfolioWeights. In Financial surveillance, Frisén M (ed). Wiley: Chichester, 2007.

49. Sonesson C, Bock D. A review and discussion of prospective statistical surveillance in public health. Journal of the Royal Statistical Society A 2003; 166:5-21.

50. Kaufmann AF, Meltzer MI, Schmid GP. The economic impact of a bioterrorist attack: Are prevention and postattack intervention programs justifiable? Emerging Infectious Diseases 1997; 3:83-94.

51. Stroup DF, Brookmeyer R, Kalsbeek WD. Public Health Surveillance in Action: A framework. In Monitoring the Health of Populations: Statistical Principles \& Methods for Public Health Surveillance, Brookmeyer R, Stroup DF (eds). Oxford University Press: Oxford, 2004. 
52. Aylin P, Best N, Bottle A, Marshall C. Following Shipman: a pilot system for monitoring mortality rates in primary care. The Lancet 2003; 362:485-491.

53. Andersson E, Bock D, Frisén M. Modeling influenza incidence for the purpose of On-Line monitoring. Statistical Methods in Medical Research 2008; 17:421-438. DOI: 10.1177/0962280206078986.

54. Frisén M, Andersson E. Semiparametric surveillance of outbreaks. Sequential Analysis 2009; 28:434-454.

55. Bock D, Andersson E, Frisén M. Statistical Surveillance of Epidemics: Peak Detection of Influenza in Sweden. Biometrical Journal 2007; 50:71-85. DOI: doi:10.1002/bimj.200610362.

56. Frisén M, Andersson E, Schiöler L. Robust outbreak surveillance of epidemics in Sweden. . Statistics in Medicine 2008:revised version submitted.

57. Andersson E, Kuhlmann-Berenzon S, Linde A, Schiöler L, Rubinova S, Frisén M. Predictions by early indicators of the progress of the influenza in Sweden. Scandinavian Journal of Public Health 2008; 36:475-482. DOI: $10.1177 / 1403494808089566$.

58. Hill GB, Spicer CC, Weatherall JAC. The computer surveillance of congenital malformations. British Medical Bulletin 1968; 24:215-218.

59. Källén B, Winberg J. Multiple malformations studied with a national register of malformations. Pediatrics 1969; 44:410-417.

60. Lawson AB, Kleinman K (eds). Spatial and Syndromic Surveillance for Public Health. Wiley: Chichester, 2005.

61. Kulldorff M, Nagarwalla N. Spatial disease clusters: Detection and inference. Statistics in Medicine 1995; 14:799-810.

62. Petzold M, Sonesson C, Bergman E, Kieler H. Surveillance in longitudinal models. Detection of intrauterine growth retardation. Biometrics 2004; 60:1025-1033.

63. Smith AF, West M. Monitoring Renal Transplants: An Application of the Multiprocess Kalman Filter. Biometrics 1983; 39:867-878.

64. Pettersson M. Monitoring a freshwater fish population: Statistical surveillance of biodiversity. Environmetrics 1998; 9:139-150.

65. Järpe E. On univariate and spatial surveillance. Ph.D Thesis. In On univariate and spatial surveillance. Ph.D Thesis, Editor (ed)^(eds). Göteborg University: City, 2000.

66. Järpe E. Surveillance, environmental. In Encyclopedia of Environmetrics, El-Shaarawi A, Piegorsh WW (eds). Wiley: Chichester, 2001.

67. Schmid W, Schöne A. Some Properties of the EWMA Control Chart in the Presence of Autocorrelation. The Annals of Statistics 1997; 25:1277-1283.

68. Kramer H, Schmid W. Control charts for time series. Nonlinear Analysis-Theory Methods \& Applications 1997; 30:4007-4016.

69. Nikiforov IV. Cumulative Sums for Detection of Changes in Random Process Characteristics. Automation and Remote Control 1979; 40:192-200.

70. Basseville M, Nikiforov I. Detection of Abrupt changes: Theory and Application. Prentice Hall: Englewood Cliffs, 1993.

71. Yashchin E. Performance of CUSUM control schemes for serially correlated observations. Technometrics 1993; 35:3752.

72. Schmid W. CUSUM control schemes for Gaussian processes. Statistical Papers 1997; 38:191-217.

73. Lai TL. Information Bounds and Quick Detection of Parameters in Stochastic Systems. IEEE Transactions on Information Theory 1998; 44:2917-2929.

74. Han D, Tsung F. The Optimal Stopping Time for Detecting Changes in Discrete Time Markov Processes. Sequential Analysis 2009; 28:115-135.

75. Borror CM, Champ CW, Rigdon SE. Poisson EWMA control charts. Journal of Quality Technology 1998; 30:352-361.

76. Kenett R, Pollak M. Data-analytic aspects of the Shiryaev-Roberts control chart: surveillance of a non-homogeneous Poisson process. Journal of Applied Statistics 1996; 23:125-137.

77. Pollak M, Siegmund D. Approximations to the Expected Sample Size of Certain Sequential Tests. The Annals of Statistics 1975; 3:1267-1282.

78. Sullivan JH, Jones LA. A self-starting control chart for multivariate individual observations. Technometrics 2002; 44:24-33.

79. Krieger AM, Pollak M, Yakir B. Surveillance of a simple linear regression. Journal of the American Statistical Association 2003; 98:456-469.

80. Sweet AL. Using Coupled Ewma Control Charts For Monitoring Processes With Linear Trends. IIE Transactions 1988; 20:404-408.

81. Zou C, Liu Y, Wang Z. Comparisons of control schemes for monitoring the means of processes subject to drifts. Metrika 2009; 70:141-163.

82. Chang JT, Fricker RD. Detecting when a monotonically increasing mean has crossed a threshold. Journal of Quality Technology 1999; 31:217-234.

83. Buehler JW, Berkelman R, Hartley D, Peters C. Syndromic surveillance and bioterrorism-related epidemics. Emerging Infectious Diseases 2003; 9:1197-1204.

84. Frisén M. Unimodal regression. The Statistician 1986; 35:479-485.

85. Frisén M, Andersson E, Pettersson K. Semiparametric estimation of outbreak regression. Statistics 2010; 44:107-117. DOI: 10.1080/02331880903021484.

86. Frisén M. Statistical Surveillance of Business Cycles. Research report 1994:1. Department of Statistics, Göteborg University, Sweden, 1994.

87. Andersson E. Monitoring cyclical processes - a nonparametric approach. Journal of Applied Statistics 2002; 29:973990. 
88. Sonesson C, Frisén M. Multivariate surveillance. In Spatial surveillance for public health, Lawson A, Kleinman K (eds). Wiley: New York, 2005.

89. Joner JMD, Woodall WH, Reynolds Jr MR, Fricker RD. A One-sided MEWMA Chart for Health Surveillance. Quality and Reliability Engineering International 2008; 24:503-518.

90. Knoth S, Schmid W. Monitoring the mean and the variance of a stationary process. Statistica Neerlandica 2002; 56:77100.

91. Fuchs C, Kenett R. Multivariate Quality Control. Theory and Application. Marcel Dekker: NY, 1998.

92. Bersimis S, Psarakis S, Panaretos J. Multivariate statistical process control charts: an overview. Quality and Reliability Engineering International 2007; 23:517-543.

93. Tsung F. Improving Automatic-Controlled Process Quality Using Adaptive Principal Component Monitoring. Quality and Reliability Engineering International 1999; 15:135-144.

94. Wessman P. Some Principles for surveillance adopted for multivariate processes with a common change point. Communications in Statistics. Theory and Methods 1998; 27:1143-1161.

95. Frisén M, Andersson E, Schiöler L. Sufficient reduction in multivariate surveillance. Communications in Statistics Theory and Methods 2010:to appear.

96. Schiöler L, Frisén M. Multivariate outbreak detection. Research report 2010:2. Statistical Research Unit, Department of Economics, University of Gothenburg, Sweden: Gothenburg, 2010.

97. Lowry CA, Woodall WH, Champ CW, Rigdon SE. A multivariate exponentially weighted moving average control chart. Technometrics 1992; 34:46-53.

98. Runger GC, Keats JB, Montgomery DC, Scranton RD. Improving the performance of the multivariate exponentially weighted moving average control chart. Quality and Reliability Engineering International 1999; 15:161-166.

99. Frisén M, Andersson E, Schiöler L. Evaluation of Multivariate Surveillance. Journal of Applied Statistics 2010; 37:2089-2100.

\section{Author's biography}

Marianne Frisén is professor emerita in Statistics at Statistical Research Unit, Department of Economics, University of Gothenburg, Gothenburg, Sweden. She received a PhD in Statistics from this university. She is an Elected member of the International Statistical Institute. Her main interests are in statistical surveillance, the foundations of statistical inference, robust methods, order restricted inference, and applied work. 
Research Report

2008:1 Frisén, M.

2008:2 Jonsson, $\mathrm{R}$.

2008:3 Andersson, E.

2008:4 Schiöler, L. \& Frisén, M.

2008:5 Schiöler, L.

2008:6 Schiöler, L.

2008:7 Andersson, E \& Frisén, $M$.

2009:1 Frisén, M., Andersson, E. $\quad$ Evaluation of Multivariate Surveillance \& Schiöler, L.

2009:2 Frisén, M., Andersson, E. Sufficient Reduction in Multivariate Surveillance \& Schiöler, L.

2010:1 Schiöler, L

2010:2 Schiöler, L. \& Frisén, M.

2010:3 Jonsson, R.

2010:4 Jonsson, R.

2011:1 Jonsson, R.

2011:2 Frisén, M
Introduction to financial surveillance.

When does Heckman's two-step procedure for censored data work and when does it not?

Hotelling's T2 Method in Multivariate On-Line Surveillance. On the Delay of an Alarm.

On statistical surveillance of the performance of fund managers.

Explorative analysis of spatial patterns of influenza incidences in Sweden 1999-2008.

Aspects of Surveillance of Outbreaks.

Statistiska varningssystem för hälsorisker

Modelling the spatial patterns of influenza incidence in Sweden

Multivariate outbreak detection

Relative Efficiency of a Quantile Method for Estimating Parameters in Censored TwoParameter Weibull Distributions

A CUSUM procedure for detection of outbreaks in Poisson distributed medical health events Simple conservative confidence intervals for comparing matched proportions

On multivariate control charts 